\title{
A NEW SUBCLASS OF COMPLEX HARMONIC FUNCTIONS
}

\author{
SiBel YalçIN AND Metin ÖZTÜRK
}

\begin{abstract}
Complex valued harmonic functions that are univalent and sense preserving in the unit disk $U$ can be written in the form $f=h+\bar{g}$, where $h$ and $g$ are analytic in $U$. In this paper, we introduce a class $H P(\alpha),(\alpha \geqslant 0)$ of functions which are harmonic in $U$. We give sufficient coefficient conditions for normalized harmonic functions in $H P(\alpha)$. These conditions are also shown to be necessary when the coefficients are negative. This leads to distortion bounds and extreme points.
\end{abstract}

Mathematics subject classification (2000): 30C45, 31A05.

Key words and phrases: Harmonic functions, univalent functions, extreme points, distortion bounds.

\section{REFERENCES}

[1] Clunie, J. And Sheil-Small, T. Harmonic Univalent Functions, Ann. Acad. Sci. Fenn. Ser. A I Math. 9 (1984) 3-25.

[2] Ruscheweyh, St., Neighborhoods of univalent functions, Proc. Amer. Math. Soc. 81 (1981), 521-528.

[3] Silverman, H., Harmonic univalent functions with negative coefficients, J. Math. Anal. Appl. 220 (1998), 283-289. 